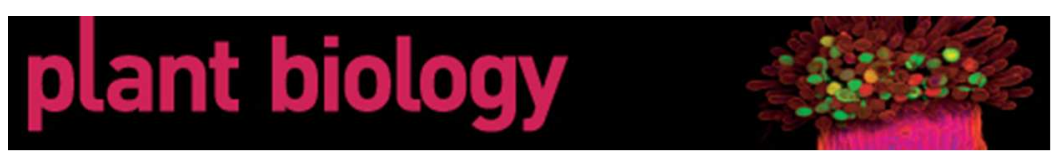

\title{
Temperature fine-tunes Mediterranean Arabidopsis thaliana life-cycle phenology geographically
}

\begin{tabular}{|r|l|}
\hline Journal: & Plant Biology \\
\hline Manuscript ID & PlaBio-2016-10-0400-RP.R2 \\
\hline Manuscript Type: & Research Paper \\
\hline Date Submitted by the Author: & n/a \\
\hline Complete List of Authors: & $\begin{array}{l}\text { Marcer, Arnald; Universitat Autonoma de Barcelona Centre de Recerca } \\
\text { Ecologica i Aplicacions Forestals } \\
\text { Vidigal, Deborah; Wageningen Universiteit } \\
\text { James, Patrick; Universite de Montreal } \\
\text { Fortin, Marie-Josee; University of Toronto } \\
\text { Mendez-Vigo, Belen; CSIC, Genética Molecular de Plantas; } \\
\text { Hilhorst, Henk W.M.; Wageningen University, Wageningen Seed Lab, Lab of } \\
\text { Plant Physiology } \\
\text { Bentsink, Leonie; Wageningen University, Laboratory of Plant Physiology; } \\
\text { Wageningen University, PSG } \\
\text { Alonso-Blanco, Carlos; Centro Nacional de Biotecnologia } \\
\text { Picó, Xavier; CSIC, EBD }\end{array}$ \\
\hline Keyword: & $\begin{array}{l}\text { adaptive evolution, environmental gradients, flowering time, seed } \\
\text { dormancy, sliding window analysis, geographically weighted regressions }\end{array}$ \\
\hline
\end{tabular}


1 RESEARCH PAPER

2

3 Temperature fine-tunes Mediterranean Arabidopsis

4 thaliana life-cycle phenology geographically

6 A. Marcer ${ }^{1,2}$, D. S. Vidigal ${ }^{3}$, P. M. A. James ${ }^{4}$, M.-J. Fortin ${ }^{5}$, B. Méndez-Vigo ${ }^{6}$,

7 H. W. M. Hilhorst ${ }^{3}$, L. Bentsink ${ }^{3}$, C. Alonso-Blanco ${ }^{6}$ \& F. X. Picó ${ }^{7}$

8

91 CREAF, Cerdanyola del Vallès, 08193 Spain

102 Univ. Autònoma de Barcelona, Cerdanyola del Vallès, 08193 Spain

113 Wageningen Seed Lab, Laboratory of Plant Physiology, Wageningen University, 6708 PB

12 Wageningen, The Netherlands

134 Département de Sciences Biologiques, Université de Montréal, C.P. 6128, succursale

14 Centre-ville, Montréal, QC H3C 3J7, Canada

155 Department of Ecology and Evolutionary Biology, University of Toronto, Toronto

16 (Ontario), M5S 3G5, Canada

176 Departamento de Genética Molecular de Plantas, Centro Nacional de Biotecnología (CNB),

18 Consejo Superior de Investigaciones Científicas (CSIC), Madrid 28049, Spain

197 Departamento de Ecología Integrativa, Estación Biológica de Doñana (EBD), Consejo

20 Superior de Investigaciones Científicas (CSIC), Sevilla 41092, Spain 
22 Running head: life-cycle phenology of Arabidopsis thaliana

23

24 Corresponding author: F. Xavier Picó, Departamento de Ecología Integrativa, Estación

25 Biológica de Doñana (EBD), Consejo Superior de Investigaciones Científicas (CSIC), Av.

26 Américo Vespucio s/n, 41092 Sevilla, Spain. Tel.: +34 95446 6700. E-mail:

$27 \quad x p i c o @$ ebd.csic.es

28

29 Keywords: adaptive evolution, environmental gradients, flowering time, geographically

30 weighted regressions, seed dormancy, sliding window analysis 


\section{ABSTRACT}

33 - To understand how adaptive evolution in life-cycle phenology operates in plants, we need

34 to unravel the effects of geographical variation in putative agents of natural selection on

35 life-cycle phenology by considering all key developmental transitions and their co-

36 variation patterns. We address this goal by quantifying the temperature-driven and

37 geographically varying relationship between seed dormancy and flowering time in the

38 annual Arabidopsis thaliana across the Iberian Peninsula.

- We used data on genetic variation in two major life-cycle traits, seed dormancy (DSDS50) and flowering time (FT), in a collection of $300 \mathrm{~A}$. thaliana accessions from the Iberian Peninsula. The geographically varying relationship between life-cycle traits and minimum temperature, a major driver of variation in DSDS50 and FT, was explored with geographically weighted regressions. The environmentally varying correlation between DSDS50 and FT was analysed by means of sliding window analysis across a minimum temperature gradient.

- The maximum local adjustments between minimum temperature and life-cycle traits were obtained in south-western Iberian Peninsula, an area with the highest minimum temperatures. In contrast, in off-south-western locations, the effects of minimum temperature on DSDS50 were rather constant across the region, whereas those of minimum temperature on FT were more variable with peaks of strong local adjustments of GWR models in central and north-western Spain. Sliding window analysis identified a minimum temperature turning point in the relationship between DSDS50 and FT around a minimum temperature of $7.2^{\circ} \mathrm{C}$. Above this minimum temperature turning point, the variation in the FT/DSDS50 ratio became rapidly constrained and the negative correlation between FT and DSDS50 did not increase any further with increasing minimum temperatures. 
57 - South-western Iberian Peninsula emerges as an area where variation in life-cycle

58 phenology appears to be restricted by the duration and severity of the hot summer

59 drought. The temperature-driven varying relationship between DSDS50 and FT detected

60 the environmental boundaries for the co-evolution between FT and DSDS50 in $A$.

61 thaliana. In a context of global warming, we conclude that the A. thaliana phenology

62 from south-western Iberian Peninsula, determined by early flowering and deep seed

63 dormancy, might become the most common life-cycle phenotype for this annual plant in

64 the region. 


\section{INTRODUCTION}

Pronounced environmental heterogeneity in soil fertility, climate seasonality and fire frequency typically characterises Mediterranean-climate regions. Broad agreement exists that environmental heterogeneity has chiefly accounted for the high regional plant diversity observed in these scarce climate regions, i.e. almost $20 \%$ of the known vascular plant species occur in less than 5\% of the Earth's surface (Cowling et al. 1996; Lobo et al. 2001). In addition, the Quaternary glacial history of some Mediterranean-climate regions, e.g. the Mediterranean Basin, has also played a very important role in structuring their current patterns of plant biodiversity (Comes and Kadereit 1998; Hewitt 1999; Hughes et al. 2006; Médail and Diadema 2009). As a result of such inherent environmental heterogeneity, the agents of natural selection are assumed to have been acting on a fine scale, which is also regarded as an additional explanation for the high plant diversity of these regions (Cowling et al. 1996). Thus, Mediterranean-climate regions are appropriate natural scenarios to undertake evolutionary studies on the processes underlying plant differentiation.

One of these evolutionary processes deals with the adaptive adjustment of life-cycle phenology, i.e. the seasonal timing of developmental events spanning from seed to seed, to heterogeneous environments (Rathcke and Lacey 1985; Donohue 2005; Wilczek et al. 2009; Huang et al. 2010; Chiang et al. 2013; Burghardt et al. 2015). Life-cycle phenology must be regarded as a composite trait influenced by the interaction between genetic and environmental factors and whose adaptive variation enhances long-term population fitness. Variation in the life-cycle phenology components with the highest impact on fitness, i.e. the timing of seed germination and the timing of reproduction, are expected to be related to the variation exhibited by their putative agents of natural selection (Caicedo et al. 2004; Stinchcombe et al. 2004; Lempe et al. 2005; Shindo et al. 2005; Méndez-Vigo et al. 2011; Kronholm et al. 2012; Manzano-Piedras et al. 2014; Vidigal et al. 2016). As climate broadly acts as a major 
selective pressure on life-cycle phenology, adaptive patterns of variation in continuous populations are commonly captured by distinctive geographical clines, revealing the interplay between life-cycle phenology, genetic loci that govern fitness-related life-cycle traits, and climatic factors (Aranzana et al. 2005; Atwell et al. 2010; Fournier-Level et al. 2011, 2013; Hancock et al. 2011; Méndez-Vigo et al. 2011; Savolainen et al. 2013; Vidigal et al. 2016). It is becoming increasingly accepted that the evolutionary implications of variation in life-cycle phenology cannot be fully understood if its components are considered in isolation (Toorop et al. 2012; Debieu et al. 2013; Burghardt et al. 2016). As a matter of fact, the success of an organism when facing heterogeneous or novel environments depends on its ability to coordinate adaptive shifts in fitness-related traits that are closely correlated with each other (Montesinos-Navarro et al. 2011; Fischer et al. 2016). Recent studies on the annual plant Arabidopsis thaliana indeed showed the influence that flowering time, which strongly determines seed maturation conditions and post-dispersal temperatures, has on seed dormancy induction and germination behaviour (Donohue 2005, 2009, 2014; Finch-Savage and Leubner-Metzger 2006; Chiang et al. 2011; Springthorpe and Penfield 2015; Burghardt et al. 2015, 2016). Hence, seed dormancy and flowering time, given their impact on fitness, are expected to be tightly correlated, eventually determining adaptive variation in life-cycle phenology.

Such a correlation has recently been found in A. thaliana, indicating that seed dormancy and flowering time are negatively correlated at both regional (Vidigal et al. 2016) and global (Debieu et al. 2013) scales. Interestingly, patterns of co-variation between seed dormancy and flowering time appear to vary geographically and independently of major genetic population structure. This supports the view that geographical patterns of variation in life-cycle phenology are adaptive (Chuine 2010; Debieu et al. 2013) and that correlations between fitness-related traits need not be constant over space and probably over time (Fischer 
115 et al. 2016). Nevertheless, gaining and in-depth understanding of the evolutionary potential of

116 co-variation between seed dormancy and flowering time might be more complicated than

117 expected. For example, Debieu et al. (2013) used a set of 112 A. thaliana accessions to show

118 how vegetative growth, flowering time and seed dormancy followed a latitudinal cline across

119 Europe and how co-variation patterns between these traits differed between North and South

120 Europe. However, only latitude appeared to have some explanatory power in their study,

121 explaining only up to $5 \%$ and $11 \%$ of the observed variation in seed dormancy and flowering

122 time, respectively (Debieu et al. 2013). In contrast, Vidigal et al. (2016), using a collection of

123300 A. thaliana accessions exclusively from the Iberian Peninsula, observed that early-

124 flowering accessions with very high seed dormancy mostly occurred in south-western Iberian

125 Peninsula. In this case, the high number of geo-referenced accessions allowed the

126 identification of minimum temperature as a major driver of variation in these traits. Average

127 annual minimum temperature alone explained up to $44.8 \%$ and $39.5 \%$ of the genetic variation

128 in seed dormancy and flowering time, respectively (Vidigal et al. 2016). Overall, these results

129 stress the value of high-density regional collections of natural accessions to study large-scale

130 variation in A. thaliana's life-cycle phenology. However, regional patterns can vary

131 substantially across the species' distribution range, which eventually determine the density of

132 populations needed to detect such patterns.

133 The main goal of this study is to reveal and quantify the temperature-driven and

134 geographically varying relationship between seed dormancy and flowering time in $A$. thaliana

135 across the Iberian Peninsula in West Mediterranean Basin, which is the $A$. thaliana's

136 distribution area known to harbour the largest regional genomic diversity (Picó et al. 2008;

137 Cao et al. 2011; Weigel 2012; Brennan et al. 2014; The 1001 Genomes Consortium 2016).

138 We use recent data on genetic variation in seed dormancy and flowering time for the 300

139 Iberian A. thaliana accessions mentioned above (Vidigal et al. 2016). We hypothesise that the 
140 negative correlation exhibited between seed dormancy and flowering time is spatially

141 heterogeneous across the Iberian Peninsula. It is already known that temperature strongly

142 shapes phenotypic variation in Iberian A. thaliana with warmer environments selecting for

143 early flowering (Méndez-Vigo et al. 2011; Manzano-Piedras et al. 2014) and high seed

144 dormancy (Vidigal et al. 2016). Beyond this general trend, however, we ignore how the

145 species fine-tunes life-cycle phenology across the region, since temperature sharply changes

146 along altitudinal and latitudinal clines. This requires a spatially-explicit approach to quantify

147 the effects of temperature variation, not only on seed dormancy and flowering time separately,

148 but on the relationship between these two major components. This knowledge will help better

149 understand how adaptive evolution in life-cycle phenology operates in plants (Debieu et al.

150 2013) as well as inferring how plants will manage to keep up with global climate change by

151 modifying their life-cycle phenology (Wilczek et al. 2014).

153 MATERIAL AND METHODS

\section{Source accessions}

155 The 300 Iberian $A$. thaliana accessions are part of a long-term project to generate a permanent

156 collection of natural populations from south-western Mediterranean Basin across Spain,

157 Portugal and Morocco for the integrative and comprehensive study of the species'

158 evolutionary ecology and functional genetics (Picó et al. 2008; Méndez-Vigo et al. 2011;

159 Brennan et al. 2014; Manzano-Piedras et al. 2014; Marcer et al. 2016; Vidigal et al. 2016).

160 For this study, we surveyed natural populations across the entire Iberian Peninsula (latitude

161 range $=36.52^{\circ} \mathrm{N}-43.40^{\circ} \mathrm{N}$, longitude range $=3.19^{\circ} \mathrm{E}-8.54^{\circ} \mathrm{W} ;$ altitude range $=1-2662$

162 m.a.s.l.; between-population distance range $=1-1042 \mathrm{~km}$ ) uninterruptedly between 2000 and

163 2010. We collected seed from several individuals per population depending on population

164 size. A few months after the field season of every sampling year, individuals were multiplied 
165 by the single seed descent method in controlled conditions in the glasshouses at the Centro

166 Nacional de Biotecnología (Madrid, ES) and stored in dry conditions in cellophane bags at

167 room temperature in darkness. When possible, accessions were chosen based on their

168 flowering time and/or vernalization requirement during the multiplication experiments. This

169 is important for our study as we selected one individual with a common phenotype within its

170 population, increasing the odds of using those phenotypes best suited to their local

171 environments. By selecting a common phenotype per population, we are also buffering the

172 effects, if any, of changing environmental conditions during the sampling period (2000-2010)

173 on life-cycle traits. Here, we assume that temporal changes in environmental conditions

174 during this period of time have not substantially affected population mean values of major

175 life-cycle traits of interest, as suggested by preliminary data (F.X. Picó et al., unpublished

176 material). Study accessions were genetically different from each other based on 250

177 polymorphic genome-wide neutral SNPs (Manzano-Piedras et al. 2014). In 2013, all

178 accessions were multiplied again in the greenhouses at Wageningen University (Wageningen,

$179 \mathrm{NL}$ ) to synchronise their seed production, which is mandatory to obtain accurate seed

180 dormancy estimates (Vidigal et al. 2016).

181

182 Life-cycle phenology

183 Arabidopsis thaliana's life-cyle phenology was characterised by variation in two major life-

184 cycle traits: seed dormancy and flowering time. For each accession, seed dormancy was

185 quantified as DSDS50, i.e. days of seed dry storage required to reach $50 \%$ of germination (see

186 He et al. 2014 for details on its calculation), whereas flowering time (FT hereafter) was

187 quantified as the number of days from the planting date until the anthesis of the first flower.

188 To estimate DSDS50, vernalized mother plants were first grown in standard conditions in a

189 glasshouse $\left(20^{\circ} \mathrm{C}\right.$ day $/ 18^{\circ} \mathrm{C}$ night; $16 \mathrm{~h}$ light: $8 \mathrm{~h}$ darkness; $70 \%$ relative humidity) to 
190 synchronise seed harvest among all accessions. Subsequently, seed was used to estimate

191 DSDS50 in an incubator at $22^{\circ} \mathrm{C}$ and constant light during 5 days (see Vidigal et al. 2016). FT

192 was estimated in a growth chamber at $21^{\circ} \mathrm{C}$ with a long-day photoperiod $(16 \mathrm{~h}$ light: $8 \mathrm{~h}$

193 darkness; see Méndez-Vigo et al. 2011). Experiments to estimate DSDS50 and FT lasted for a

194 maximum of 559 and 220 days, respectively (Méndez-Vigo et al. 2011; Vidigal et al. 2016).

195 Both seed dormancy and flowering time were estimated under controlled laboratory

196 conditions, providing the quantitative genetic component for both traits.

198 Minimum temperature data

199 Previous studies indicated that minimum temperature is the main contributing factor to the

200 observed patterns of variation in DSDS50 and FT in Iberian A. thaliana far above other

201 environmental factors (Méndez-Vigo et al. 2011; Manzano-Piedras et al. 2014; Vidigal et al.

202 2016). Given the predominant role of minimum temperature shaping patterns of phenotypic

203 variation in Iberian A. thaliana, for the sake of clarity and simplicity we used the average

204 annual minimum temperature (minimum temperature hereafter) as the explanatory variable in

205 all analyses. Additional analyses using variables related to precipitation, vegetation and soil,

206 alone or in combination with minimum temperature, neither improved the outcomes nor

207 added any value to the interpretation of the resulting patterns in a significant way (results not

208 shown). Minimum temperature from every population was obtained from the Digital Climatic

209 Atlas of the Iberian Peninsula (Ninyerola et al. 2000). Minimum temperature data came from

210 a spatial interpolation using temperature records from a total of 3608 meteorological stations

211 across Portugal and Spain during the period 1950-1999. Hence, although our A. thaliana

212 populations were sampled between 2000 and 2010, the minimum temperature data used in

213 this study reflect the environmental conditions where A. thaliana has probably been occurring

214 and adapting for long time in the Iberian Peninsula. 


\section{Statistical analyses}

217 The hypothesised geographically varying relationship between life-cycle phenology traits and 218 minimum temperature was explored with geographically weighted regression (GWR) models 219 (Fotheringham et al. 2002) using SAM software (Rangel et al. 2010). First, we performed a 220 GWR to test the effect of minimum temperature on DSDS50 and another GWR to test the 221 effect of minimum temperature on FT. GWR is a spatially-explicit explanatory regression 222 where regression parameters are estimated at each sampled location $i$ as follows:

223

224 225

226

$y_{i}=\beta\left(v_{i}, v_{i}\right) x_{i}+\varepsilon_{i}$,

where $y_{i}$ is the dependent variable, $x_{i}$ is the independent variable, $\varepsilon_{i}$ is the Gaussian error, $\left(v_{i}, v_{i}\right)$ are the geographical coordinates, and $\beta\left(v_{i}, v_{i}\right)$ is the GWR varying coefficient at each location $i$. GWR performed a local regression at each of the 300 locations by taking a fraction $(10-15 \%)$ of the neighbouring locations into account using an optimal bandwidth size. For each location, the selected bandwidth was optimised by using a Gaussian spatial weighting function minimising the Akaike information criterion (AICc). This procedure generally enhances the goodness-of-fit of GWR by setting the optimal number of neighbouring locations to perform local regressions as well as by controlling for border and sampling effects. GWR and OLS (ordinary least squares) results were also compared with an ANOVA to check that the explanatory power of GWR was better than that of OLS. The lack of autocorrelation of the residuals was checked with a spatial correlogram plotting Moran's $I$ values of GWR residuals. Thus, GWR provided local estimates of the adjustment of DSD50 and FT values to minimum temperatures in Iberian $A$. thaliana populations, i.e. local adjusted pseudo- $R^{2}$ values. 
After assessing the relationship between minimum temperature and DSDS50 and FT

in two separate GWR models, we uncovered the environmentally varying correlation between DSDS50 and FT by means of sliding window analysis (as in Debieu et al. 2013). Sliding window analyses are used to display the dynamic relationship between variables across a continuous space. In this study, we used a temperature sliding window to account for the covariation between life-cycle traits across a minimum temperature gradient. We selected a temperature sliding window instead of a geographical sliding window because minimum temperature does a better job than geography in characterising the environment of Iberian $A$. thaliana populations. For example, geographically close populations can exhibit pronounced differences in minimum temperature if they are located along the same altitudinal gradient (Montesinos et al. 2009; Méndez-Vigo et al. 2011). Hence, all 300 A. thaliana populations were first ranked according to their minimum temperatures (range $=-0.7^{\circ}-13.0^{\circ} \mathrm{C}$ ). For every population along the minimum temperature gradient and regardless of its geographical location, we sub-sampled all populations whose minimum temperatures fell within a temperature interval around it. We tested different temperature breadths and eventually selected a temperature interval of $7^{\circ} \mathrm{C}$ centred on the minimum temperature characterising each population. The temperature breadth determines the number of populations falling within the temperature interval as well as the truncation of the sampling window for those populations at the low and high ends of the temperature gradient. Consistent patterns were detected with narrower temperature breadths (results not shown) but the chosen one optimised the patterns observed.

For each of the 300 values of the minimum temperature gradient, we computed the correlation between DSDS50 and FT as well as the mean and SD of the FT/DSDS50 ratio using all the accessions falling within the temperature interval of $7^{\circ} \mathrm{C}$ (mean $\pm \mathrm{SD}$ number of sampling accessions per minimum temperature $=194.6 \pm 53.1)$. It must be noted that we used 
265 more than 100 accessions in $93 \%$ of the analyses ( 279 of 300 analyses). The FT/DSDS50

266 ratio provided an integrated measure of variation between the two life-cycle traits because

267 accessions with early flowering times and high seed dormancy had low FT/DSDS50 ratios,

268 whereas late flowering accessions with low seed dormancy exhibited high FT/DSDS50 ratios.

269 The significance of the correlations between DSDS50 and FT for every minimum temperature

270 was tested with the Dutilleul's modified $t$ test, using the R package SpatialPack (Osorio and

271 Vallejos 2014), to take any spatial autocorrelation in the data into account. The Dutilleul's

272 modified $t$ test corrects the variance of the test statistic and the degrees of freedom according

273 to the extent of spatial autocorrelation of each variable of the correlation (Dutilleul et al.

274 1993). Our large sample size $(N=300)$ ensures a high accuracy of the results, as required in

275 spatially-explicit statistical tests (Legendre et al. 2002).

276

277 RESULTS

278 The geographically varying relationship between life-cycle phenology traits, i.e. DSDS50 and

279 FT, and minimum temperature was explored with two separate geographically weighted

280 regression (GWR) models. For DSDS50 and minimum temperature, the GWR results showed

281 that local models based on GWR performed better than the global model based on the

282 classical ordinary least squares (OLS) regression model $\left(F_{5.9,292.1}=6.68, P<0.0001\right.$;

283 ANOVA; adjusted $R^{2}=0.41$ and 0.34 for GWR and OLS regression, respectively). All local

284 models were significant $(P<0.001$ in all cases $)$ and all local standardised coefficients of

285 regression were positive (range of $r=0.33-0.90$ ), indicating that minimum temperature and

286 DSDS50 were positively correlated in all cases, i.e. accessions from warmer locations

287 exhibited higher seed dormancy. The local adjusted pseudo- $R^{2}$ values (range of pseudo- $R^{2}=$

$2880.18-0.50$ ) clearly increased along a north-south latitudinal gradient reaching the highest

289 values in south-western Iberian Peninsula (Fig. 1A). 
For FT and minimum temperature, the GWR results also showed that local models

291 performed better than the global OLS model $\left(F_{9.4,288.6}=8.90, P<0.0001\right.$; ANOVA; adjusted

$292 R^{2}=0.49$ and 0.36 for GWR and OLS regression, respectively). All local models were

293 significant $(P<0.003$ in all cases $)$ and all local standardised coefficients of regression were

294 negative (range of $r=-0.31--1.00$ ). Therefore, minimum temperature and FT were

295 negatively correlated in all cases, i.e. accessions from warmer locations flowered earlier. The

296 local adjusted pseudo- $R^{2}$ values for FT were more variable (range of pseudo- $R^{2}=0.13-0.65$ )

297 and exhibited more geographical complexity than those estimated for DSDS50 (Fig. 1B). In

298 particular, south-western Iberian Peninsula was again an area where the adjustment between

299 minimum temperature and FT peaked, but strong adjustment peaks in other nuclei of

300 accessions in central and north-western Spain were also detected (Fig. 1B).

301 The environmentally varying relationship between DSDS50 and FT was analysed with

302 a temperature sliding window analysis. The results depicted the co-variation between DSD50

303 and FT along a minimum temperature gradient. The mean $( \pm \mathrm{SD})$ of the FT/DSDS50 ratio

304 computed from all accessions falling within the temperature interval of $7^{\circ} \mathrm{C}$ along the

305 minimum temperature gradient varied from a low of $0.26 \pm 0.21$, i.e. accessions with early

306 flowering time and high seed dormancy, to a high of $2.10 \pm 0.79$, i.e. accessions with late

307 flowering time and low seed dormancy (Fig. 2A). As expected, the mean of the FT/DSDS50

308 ratio progressively decreased with increasing minimum temperatures (Fig. 2A). In contrast,

309 the slope of the relationship between minimum temperature and the SD of the FT/DSDS50

310 ratio exhibited a turning point at a minimum temperature around $7.2^{\circ} \mathrm{C}$ : the $\mathrm{SD}$ of the

311 FT/DSDS50 ratio decreased faster with minimum temperatures above this turning point (Fig.

312 2B).

313 The temperature sliding window analysis also indicated that the correlation between

314 FT and DSDS50 was not significant for populations with minimum temperatures below $3.6^{\circ} \mathrm{C}$ 
315 and above $12.3^{\circ} \mathrm{C}(N=42$ out of 300 ; Fig. $2 \mathrm{C})$. In between these two thresholds, the

316 correlation between FT and DSDS50 along the minimum temperature axis was significant and

317 negative, but progressively increased with increasing minimum temperatures around $7.3^{\circ} \mathrm{C}$

318 (range of $r$ values before the turning point $=-0.18--0.39$; Fig. 2C). From that minimum

319 temperature onwards, the significant correlation between FT and DSDS50 first had relatively

320 constant coefficients close to the maximum observed value to end with a more variable

321 behaviour with increasing minimum temperature (range of $r$ values after the turning point $=$ -

$3220.30--0.41$; Fig. 2 C). It is worth noting that the turning point in the relationship between FT

323 and DSDS50 along a minimum temperature gradient was also observed with additional

324 sliding window analyses depicting the correlation between each life-cycle trait and minimum

325 temperature along the minimum temperature gradient (Fig. S1). These results showed that the

326 correlation between DSDS50 and minimum temperature and between FT and minimum

327 temperature also reached a major turning point very close to those reported above.

328 The representation of two groups of populations, whose minimum temperature is

329 above and below the turning point for the correlation between FT and DSDS50, onto a

330 minimum temperature map, provides hints on the geographical and ecological differentiation

331 of A. thaliana governed by minimum temperature (Fig. 3). In particular, A. thaliana

332 populations with minimum temperatures below the turning point are mostly located in the

333 northern half of the Iberian Peninsula, including the main mountain ranges (e.g. Pyrenees,

334 Cantabrian, Central and Iberian ranges), the northern plateau, and Sierra Nevada in South

335 Spain with the highest mountain of the Iberian Peninsula (Fig. 3). In contrast, A. thaliana

336 populations with minimum temperatures above the turning point occur in the southern half of

337 the Iberian Peninsula as well as in coastal locations around the region (Fig. 3). As a result, the

338 two groups of $A$. thaliana populations occurring below $(N=191)$ and above $(N=109)$ the

339 minimum temperature turning point for the correlation between FT and DSDS50 significantly 
340 differed in altitude (mean $\pm \mathrm{SE}$ altitudes $=973.7 \pm 24.0$ and $459.4 \pm 24.1$ m.a.s.l. for the

341 former and the latter, respectively; $F_{1,298}=198.61, P<0.0001$; one-way ANOVA). In

342 addition, higher elevation populations with minimum temperatures below the turning point

343 also exhibited a higher percentage of wild habitat than lower elevation populations with

344 minimum temperatures above the turning point (mean \pm SE percentages of wild habitat $=64.4$

$345 \pm 2.6$ and $45.3 \pm 3.7 \%$ for the former and the latter, respectively; $F_{1,298}=18.95, P<0.0001$;

346 one-way ANOVA). This makes sense as human activity with more dramatic impacts on

347 natural plant communities has traditionally been concentrated at lower elevations. In contrast,

348 the two groups of populations did not differ in total annual precipitation, mean annual solar

349 radiation, or soil $\mathrm{pH}(P>0.10$ in all cases; one-way ANOVA). Overall, these results stress the

350 explanatory power of minimum temperature for variation in life-cycle phenology in Iberian $A$.

\section{1 thaliana.}

\section{DISCUSSION}

354 Mediterranean-climate regions represent outstanding natural scenarios to address multitude of

355 evolutionary issues in plants. Adaptive variation in life-cycle phenology is particularly

356 appropriate because major events of life-cycle phenology are markedly influenced by climatic

357 factors, whose seasonality, in fact, defines the Mediterranean climate. It is clear that we need

358 to unravel the effects of climatic factors, not only on life-cycle traits separately, but on the

359 life-cycle phenology as a whole (Post et al. 2008; Debieu et al. 2013; Burghardt et al. 2016).

360 This is supported by increasingly abundant studies on A. thaliana as an evolutionary model

361 system, focused on the tight interplay between flowering time and seed germination

362 (Donohue 2005, 2009, 2014; Finch-Savage and Leubner-Metzger 2006; Chiang et al. 2011;

363 Toorop et al. 2012; Debieu et al. 2013; Wilczek et al. 2014; Springthorpe and Penfield 2015;

364 Burghardt et al. 2015, 2016) as well as on the effects of seasonal environmental variation on 
365 key developmental-timing genes affecting multiple developmental transitions (Chiang et al.

366 2009; Atwell et al. 2010; Chen et al. 2014; Huo et al. 2016). Nevertheless, the separate

367 analyses of the geographically varying relationship between minimum temperature and $A$.

368 thaliana's life-cycle traits still provide valuable elements that are worth considering. For

369 example, the GWR results indicated that south-western Iberian Peninsula represents an area

370 with strong local adjustments for both FT and DSDS50 (Fig. 1). This confirms the capital role

371 of minimum temperatures in shaping up life-cycle phenology towards early flowering time

372 and strong seed dormancy as the environment becomes warmer (Méndez-Vigo et al. 2011;

373 Manzano-Piedras et al. 2014; Vidigal et al. 2016).

374 Interestingly, our analyses indicated that for the rest of the Iberian Peninsula there

375 were notorious differences in the geographically varying effect of minimum temperature on

376 each of the study life-cycle traits. On the one hand, the positive relationship between

377 minimum temperature and DSDS50 remained rather moderate and homogeneous for

378 accessions from off-south-western locations (Fig. 1A). Overall, these results suggest that

379 minimum temperature increases its intensity as an agent of natural selection for DSDS50

380 towards south-western Iberian Peninsula, i.e. an area with the highest minimum temperatures.

381 Based on this, we hypothesise that the windows of opportunity for germination also follow a

382 north-south gradient, becoming narrower towards south-western Iberian Peninsula as a result

383 of the longer and more severe summer droughts in that area. Although field demographic

384 studies from northern $A$. thaliana populations indicated that germination peaks in early/mid

385 autumn, and to a lesser extent between late winter and early spring (Montesinos et al. 2009;

386 Picó 2012), we still lack germination data from southern populations. Therefore, further field

387 work is needed to quantify in detail the geographical patterns of variation in seed germination

388 behaviour in natural $A$. thaliana populations across the warmest area of the Iberian Peninsula

389 to test this hypothesis. 
On the other hand, the negative relationship between minimum temperature and FT in

391 off-south-western locations was far more variable, with additional areas of pronounced local

392 adjustments of the GWR models, especially in central and north-western Spain (Fig. 1B). In

393 this case, orography might explain these results because central and north-western Spain are

394 mountainous areas with remarkable altitudinal gradients, which imply a rapid change in

395 ecological conditions over relatively short distances. As a matter of fact, altitudinal gradients

396 represent an important source of phenotypic and adaptive variation in $A$. thaliana in different

397 regions across its distribution range (Montesinos et al. 2009; Méndez-Vigo et al. 2011;

398 Montesinos-Navarro et al. 2011; Picó 2012; Suter et al. 2014; Luo et al. 2015a, 2015b; Tyagi

399 et al. 2016; but see Günther et al. 2016). We have previously shown that decreasing

400 temperatures with increasing altitude in Iberian mountains are related to late flowering in $A$.

401 thaliana through the obligate vernalization requirement (Méndez-Vigo et al. 2011). This is a

402 flowering-inductive effect of low temperature that appears to be restricted to altitudes above

403800 m (Méndez-Vigo et al. 2011), locations with typically cooler climates that are practically

404 absent in south-western Iberian Peninsula (Marcer et al. 2016). Hence, the preponderance of a

405 vernalization requirement to trigger flowering at high altitude cool environments and the lack

406 thereof in generally warmer lowland environments illustrate the variety and complexity of the

407 genetic mechanisms underlying adaptive variation in FT in A. thaliana (Koornneef et al.

408 1998; Simpson and Dean 2002; Alonso-Blanco et al. 2009; Wilczek et al. 2009).

409 On top of these geographically varying relationships between minimum temperature

410 and life-cycle traits, in this study we took a step further towards understanding how the

411 relationship between FT and DSDS50 varied across a minimum temperature gradient. The

412 results of the temperature sliding window analysis indicated that FT and DSDS50 co-varied

413 negatively with minimum temperature, in agreement with the behaviour of each life-cycle

414 trait with minimum temperature shown in this study, as well as with previous observations 
415 (Debieu et al. 2013; Vidigal et al. 2016). The novel contribution of the sliding temperature

416 analysis is that it allowed the visualization and quantification of the heterogeneity of the

417 relationship between FT and DSDS50 along the minimum temperature gradient. Of particular

418 relevance is the existence of a minimum temperature turning point in the relationship between

419 FT and DSDS50, which has interesting ecological and evolutionary implications to better

420 understand life-cycle phenology, not only for $A$. thaliana, but for several other annuals and

421 short-lived perennials strongly relying on fast life cycles to succeed in their environments

422 (García et al. 2008).

423 For example, A. thaliana populations with minimum temperatures above the turning

424 point exhibited faster decreasing variability in the FT/DSDS50 ratio with respect to increasing

425 minimum temperature (Fig. 2B). This means that variation in $A$. thaliana's life-cycle

426 phenology becomes progressively constrained as minimum temperatures become higher. In

427 Iberian warm environments, the duration and severity of the summer drought force $A$.

428 thaliana to complete its life cycle quickly, which can only be accomplished by becoming

429 dormant during the long hot summer together with early flowering before temperatures

430 become too high again. In fact, A. thaliana populations from south-western Iberian Peninsula

431 shed seed between mid April and early May and, in the warmest locations, between late

432 February and mid March (C. Alonso-Blanco and F.X. Picó, personal observation). Such early

433 flowering and fruiting imply that the maternal and post-dispersal environments encountered

434 by seeds might also promote deeper physiological seed dormancy, ensuring late germinations

435 when the summer drought is over with the first autumn rains.

436 Another implication of the existence of a minimum temperature turning point in the

437 relationship between FT and DSDS50 involves the strength of the correlation between the two

438 life-cycle traits. The turning point identified a minimum temperature threshold from which

439 the correlation coefficient between FT and DSDS50 cannot increase any further (Fig. 2C). 
440 Hence, the temperature sliding window analysis provides a tool to detect the environmental

441 boundaries for the co-evolution between FT and DSDS50 for A. thaliana in Mediterranean

442 environments. In particular, below the minimum temperature threshold, A. thaliana shows

443 substantial phenotypic space to adjust its life-cycle phenology by progressively advancing

444 flowering time and increasing seed dormancy with increasing minimum temperature. Above

445 the minimum temperature threshold, A. thaliana might have reached the limits to adjust its

446 life-cycle phenology as described, which otherwise does not prevent the species to succeed in

447 warmer environments. Interestingly, populations characterised by minimum temperatures

448 above the turning point occur in southern Iberian Peninsula and in warmer coastal areas from

449 all over the Iberian Peninsula, whereas those below the turning point mostly occur in the

450 northern mountainous Iberian Peninsula (Fig. 3). Thus, unravelling the phenotypic limits for

451 the co-variation between key life-cycle traits and circumscribing such limits into a

452 geographical and ecological space, in our case strongly characterised by variation in

453 minimum temperature, might be more enlightening than depicting patterns of variation along

454 geographical or environmental gradients if we aim to extend our understanding of adaptive

455 evolution in plants.

456 In this study, we have shown that the genetic co-variation between flowering time and

457 seed dormancy is geographically fine-tuned by minimum temperature in Mediterranean $A$.

458 thaliana. However, it remains to be seen to what extent the timing of flowering initiation

459 affects the timing of seed germination, e.g. maternal environmental effects on seed dormancy

460 and germination patterns, or, conversely, whether the timing of seed germination affects the

461 timing of flowering initiation, e.g. environmental cues for flowering affecting rosettes from

462 seeds germinated at different times (see Donohue 2009). In any case, our results represent a

463 first step towards the development of spatially-explicit models to assess the evolutionary

464 dynamics of life-cycle phenology as well as the impact of climate change on adaptive 
465 variation in $A$. thaliana (see non-spatially-explicit recent models for $A$. thaliana in Burghardt 466 et al. 2015; Fournier-Level et al. 2016). This is important because it has been predicted that 467 Mediterranean-climate regions, and in particular the Mediterranean Basin, will be 468 dramatically affected by global climate change before the end of the century, with a 469 generalised warming and an increase of the duration and severity of the summer drought 470 (Klausmeyer and Shaw 2009; Gómez-Navarro et al. 2010; Jacobeit et al. 2014). In this 471 context, our results suggest that the phenology exhibited by south-western Iberian $A$. thaliana 472 determined by early flowering and deep seed dormancy, might become the most common life 473 cycle of this annual plant in the climate scenarios forecasted for this region.

474

475 ACKNOWLEDGEMENTS

476 We are grateful to technicians from CNB-CSIC, WUR and CREAF for lab assistance. We 477 thank two anonymous reviewers for their helpful and constructive comments on the 478 manuscript, and J. Arroyo and J.D. Thompson for the invitation to participate in this special 479 issue. F.X.P. and A.M. were funded by a grant from the Ministerio de Economía y 480 Competitividad of Spain (CGL2016-77720-P). F.X.P. acknowledges the Severo Ochoa 481 Programme for Centres of Excellence in R+D+I (SEV-2012-0262) in Spain. A.M., M.J.F. and 482 P.M.A.J. acknowledge the NEWFORESTS project (PIRSES-GA-2013-612645) from the 483 European 7FP. A.M. acknowledges the Agency for Management of University and Research 484 Grants of the Generalitat de Catalunya (2014-SGR-913). D.S.V. was funded by the 485 Technology Foundation STW (grant 11314). L.B. was funded by the VIDI program of the 486 Technology Foundation STW (grant 12951). 487

\section{REFERENCES}


Alonso-Blanco C., Aarts M.G.M., Bentsink L., Keurentjes J.J.B., Reymond M., Vreugdenhil D., Koornneef M. (2009) What has natural variation taught us about plant development, physiology, and adaptation? Plant Cell, 21, 1877-1896.

492

Aranzana M.J., Kim S., Zhao K., Bakker E., Horton M., Jakob K., et al. (2005) Genome-wide association mapping in Arabidopsis identifies previously known flowering time and pathogen resistance genes. PLoS Genetics, 1, e60.

Atwell S., Huang Y.S., Vilhjalmsson B.J., Willems G., Horton M., Li Y., et al. (2010) Genome-wide association study of 107 phenotypes in Arabidopsis thaliana inbred lines. Nature, 465, 627-631.

Brennan A.C., Méndez-Vigo B., Haddioui A., Martínez-Zapater J.M., Picó F.X., AlonsoBlanco C. (2014) The genetic structure of Arabidopsis thaliana in the south-western Mediterranean range reveals a shared history between North Africa and southern Europe. BMC Plant Biology, 14, 17.

502 Burghardt L.T., Metcalf C.J., Wilczek A.M., Schmitt J., Donohue K. (2015) Modeling the 503 influence of genetic and environmental variation on the expression of plant life cycles 504 across landscapes. American Naturalist, 185, 212-227.

505 Burghardt L.T., Metcalf C.J., Donohue K. (2016) A cline in seed dormancy helps conserve 506 the environment experienced during reproduction across the range of Arabidopsis thaliana. 507 American Journal of Botany, 103, 47-59.

508 Caicedo A.L., Stinchcombe J.R., Olsen K.M., Schmitt J., Purugganan M.D. (2004) Epistatic 509 interaction between Arabidopsis FRI and FLC flowering time genes generates a latitudinal 510 cline in a life history trait. Proceedings of the National Academy of Sciences, 101, 15670$511 \quad 15675$. 
512 Cao J., Schneeberger K., Ossowski S., Günther T., Bender S., Fitz J., et al. (2011) Whole-

513 genome sequencing of multiple Arabidopsis thaliana populations. Nature Genetics, $\mathbf{4 3}$,

$514956-963$.

515 Chen J., Tsuda Y., Stocks M., Källman T., Xu N., Kärkkäinen K., et al. (2014) Clinal

516 variation at phenology-related genes in spruce: parallel evolution in FTL2 and Gigantea?

517 Genetics, 197, 1025-1038.

518 Chiang G.C., Barua D., Kramer E.M., Amasino R.M., Donohue K. (2009) Major flowering

519 time gene, flowering locus C, regulates seed germination in Arabidopsis thaliana.

$520 \quad$ Proceedings of the National Academy of Sciences, 106, 11661-11666.

521 Chiang G.C., Bartsch M., Barua D., Nakabayashi K., Debieu M., Kronholm I., et al. (2011)

$522 D O G 1$ expression is predicted by the seed-maturation environment and contributes to

523 geographical variation in germination in Arabidopsis thaliana. Molecular Ecology, 20,

$524 \quad 3336-3349$.

525 Chiang G.C., Barua D., Dittmar E., Kramer E.M., de Casas R.R., Donohue K. (2013)

526 Pleiotropy in the wild: the dormancy gene DOG1 exerts cascading control on life cycles.

527 Evolution, 67, 883-893.

528 Chuine I. (2010) Why does phenology drive species distribution? Philosophical Transactions

529 of the Royal Society B, 365, 3149-3160.

530 Comes H.P., Kadereit J.W. (1998) The effect of Quaternary climatic changes on plant

531 distribution and evolution. Trends in Plant Science, 3, 432-438.

532 Cowling R.M., Rundel P.W., Lamont B.B., Arroyo M.K., Arianoutsou M. (1996) Plant

533 diversity in Mediterranean-climate regions. Trends in Ecology and Evolution, 11, 362-366.

534 Debieu M., Tang C., Stich B., Sikosek T., Effgen S., Josephs E., et al. (2013) Co-variation

535 between seed dormancy, growth rate and flowering time changes with latitude in

536 Arabidopsis thaliana. PLoS One, 8, e61075. 
537 Donohue K. (2005) Niche construction through phenological plasticity: life history dynamics

538 and ecological consequences. New Phytologist, 166, 83-92.

539 Donohue K. (2009) Completing the cycle: maternal effects as the missing link in plant life

540 histories. Philosophical Transactions of the Royal Society B, 364, 1059-1074.

541 Donohue K. (2014) Why ontogeny matters during adaptation: developmental niche

542 construction and pleiotropy across the life cycle in Arabidopsis thaliana. Evolution, 68 , $543 \quad 32-47$.

544 Dutilleul P. (1993) Modifying the $t$ test for assessing the correlation between two spatial

545 processes. Biometrics, 49, 305-314.

546 Finch-Savage W.E., Leubner-Metzger G. (2006) Seed dormancy and the control of

547 germination. New Phytologist, 171, 501-523.

548 Fischer E.K., Ghalambor C.K., Hoke K.L. (2016) Plasticity and evolution in correlated suites

549 of traits. Journal of Evolutionary Biology, 29, 991-1002.

550 Fotheringham A.S., Brunsdon C., Charlton M. (2002) Geographically Weighted Regression:

551 The Analysis of Spatially Varying Relationships. John Wiley \& Sons, Inc., Hoboken, NJ,

$552 \quad$ USA: $284 \mathrm{pp}$.

553 Fournier-Level A., Korte A., Cooper M.D., Nordborg M., Schmitt J., Wilczek A.M. (2011) A

554 map of local adaptation in Arabidopsis thaliana. Science, 334, 86-89.

555 Fournier-Level A., Wilczek A.M., Cooper M.D., Roe J. L., Anderson J., Eaton D., et al.

556 (2013) Paths to selection on life history loci in different natural environments across the

557 native range of Arabidopsis thaliana. Molecular Ecology, 22, 3552-3566.

558 Fournier-Level A., Perry E.O., Wang J.A., Braun P.T., Migneault A., Cooper M.D., et al.

559 (2016) Predicting the evolutionary dynamics of seasonal adaptation to novel climates in

$560 \quad$ Arabidopsis thaliana. Proceedings of the National Academy of Sciences, 113, E2812-

$561 \quad$ E2821. 
562 García M.B., Picó F.X., Ehrlén J. (2008) Life span correlates with population dynamics in

563 perennial herbaceous plants. American Journal of Botany, 95, 258-262.

564 Gómez-Navarro J.J., Montávez J.P., Jimenez-Guerrero P., Jerez S., García-Valero J.A.,

565 González-Rouco J.F. (2010) Warming patterns in regional climate change projections over

566 the Iberian Peninsula. Meteorologische Zeitschrift, 19, 275-285.

567 Günther T., Lampei C., Barilar I., Schmid K.J. (2016) Genomic and phenotypic

568 differentiation of Arabidopsis thaliana along altitudinal gradients in the North Italian Alps.

569 Molecular Ecology, 25, 3574-3592.

570 Hancock A.M., Brachi B., Faure N., Horton M.W., Jarymowycz L.B., Sperone F.G., et al.

571 (2011) Adaptation to climate across the Arabidopsis thaliana genome. Science, 334, 83-

57286.

573 He H., Vidigal D.S., Snoek L.B., Schnabel S., Nijveen H., Hilhorst H., et al. (2014)

574 Interaction between parental environment and genotype affects plant and seed performance

575 in Arabidopsis. Journal of Experimental Botany, 65, 6603-6615.

576 Hewitt G.M. (1999) Post-glacial re-colonization of European biota. Biological Journal of the

$577 \quad$ Linnean Society, 68, 87-112.

578 Huang X., Schmitt J., Dorn L., Griffith C., Effgen S., Takao S., et al. (2010) The earliest

579 stages of adaptation in an experimental plant population: strong selection on QTLS for

$580 \quad$ seed dormancy. Molecular Ecology, 19, 1335-1351.

581 Hughes P.D., Woodward J.C., Gibbard P.L. (2006) Quaternary glacial history of the

582 Mediterranean mountains. Progress in Physical Geography, 30, 334-364.

583 Huo H., Wei S, Bradford K.J. (2016) DELAY OF GERMINATION1 (DOG1) regulates both

584 seed dormancy and flowering time through microRNA pathways. Proceedings of the

$585 \quad$ National Academy of Sciences, 113, E2199-E2206. 
586 Jacobeit J., Hertig E., Seubert, S., Lutz K. (2014) Statistical downscaling for climate change

587 projections in the Mediterranean region: methods and results. Regional Environmental

588 Change, 14, 1891-1906.

589 Klausmeyer K.R., Shaw M.R. (2009) Climate change, habitat loss, protected areas and the

590 climate adaptation potential of species in Mediterranean ecosystems worldwide. PLoS One,

$591 \quad 4$, e6392.

592 Koornneef M., Alonso-Blanco C., Peeters A.J.M., Soppe W. (1998) Genetic control of

593 flowering time in Arabidopsis. Annual Review of Plant Physiology and Plant Molecular

594 Biology, 49, 345-370.

595 Kronholm I., Picó F.X., Alonso-Blanco C., Goudet J., de Meaux J. (2012) Genetic basis of

596 adaptation in Arabidopsis thaliana: local adaptation at the seed dormancy QTL DOG1.

$597 \quad$ Evolution, 66, 2287-2302.

598 Legendre P., Dale M.R.T., Fortin M.J., Gurevitch J., Hohn M., Myers D. (2002) The

599 consequences of spatial structure for the design and analysis of ecological field surveys.

$600 \quad$ Ecography, 25, 601-615.

601 Lempe J., Balasubramanian S., Sureshkumar S., Singh A., Schmid M., Weigel D. (2005)

602 Diversity of flowering responses in wild Arabidopsis thaliana strains. PLoS Genetics, $\mathbf{1}$,

$603 \quad 0109-0118$.

604 Lobo J.M., Castro I., Moreno J.C. (2001) Spatial and environmental determinants of vascular

605 plant species richness distribution in the Iberian Peninsula and Balearic Islands. Biological

606 Journal of the Linnean Society, 73, 233-253.

607 Luo Y., Widmer A., Karrenberg S. (2015a) The roles of genetic drift and natural selection in

608 quantitative trait divergence along an altitudinal gradient in Arabidopsis thaliana.

$609 \quad$ Heredity, 114, 220-228. 
610 Luo Y., Dong X., Yu T., Shi X., Li Z., Yang W., et al. (2015b) A single nucleotide deletion in 611 Gibberellin20-oxidase1 causes alpine dwarfism in Arabidopsis. Plant Physiology, 168, $612930-937$.

613 Manzano-Piedras E., Marcer A., Alonso-Blanco C., Picó F.X. (2014) Deciphering the 614 adjustment between environment and life history in annuals: lessons from a 615 geographically-explicit approach in Arabidopsis thaliana. PLoS One, 9, e87836.

616 Médail F., Diadema K. (2009) Glacial refugia influence plant diversity patterns in the 617 Mediterranean Basin. Journal of Biogeography, 36, 1333-1345.

618 Méndez-Vigo B., Picó F.X., Ramiro M., Martínez-Zapater J.M., Alonso-Blanco C. (2011)

619 Altitudinal and climatic adaptation is mediated by flowering traits and FRI, FLC and $620 \quad$ PHYC genes in Arabidopsis. Plant Physiology, 157, 1942-1955.

621 Montesinos A., Tonsor S.J., Alonso-Blanco C., Picó F.X. (2009) Demographic and genetic 622 patterns of variation among populations of Arabidopsis thaliana from contrasting native 623 environments. PLoS One, 4, e7213.

624 Montesinos-Navarro A., Wig J., Picó F.X., Tonsor S.J. (2011) Arabidopsis thaliana 625 populations show clinal variation in a climatic gradient associated with altitude. New 626 Phytologist, 189, 282-294.

627 Ninyerola M., Pons X., Roure J.M. (2000) A methodological approach of climatological 628 modelling of air temperature and precipitation through GIS techniques. International 629 Journal of Climatology, 20, 1823-1841.

630 Osorio F., Vallejos R. (2014) SpatialPack: Package for analysis of spatial data. R package 631 version 0.2-3. URL: CRAN.R-project.org/package=SpatialPack.

632 Picó F.X., Méndez-Vigo B., Martínez-Zapater J.M., Alonso-Blanco C. (2008) Natural genetic 633 variation of Arabidopsis thaliana is geographically structured in the Iberian Peninsula. $634 \quad$ Genetics, 180, 1009-1021. 
635 Picó F.X. (2012) Demographic fate of Arabidopsis thaliana cohorts of autumn- and spring636 germinated plants along an altitudinal gradient. Journal of Ecology, 100, 1009-1018.

637 Post E.S., Pedersen C., Wilmers C.C., Forchhammer M.C. (2008) Phenological sequences

638 reveal aggregate life history response to climatic warming. Ecology, 89, 363-370.

639 Rangel T.F., Diniz-Filho J.A.F., Bini L.M. (2010) SAM: A comprehensive application for 640 spatial analysis in macroecology. Ecography, 33, 46-50.

641 Rathcke B., Lacey E.P (1985) Phenological patterns of terrestrial plants. Annual Review of 642 Ecology, Evolution and Systematics, 16, 179-214.

643 Savolainen O., Lascoux M., Merilä J. (2013) Ecological genomics of local adaptation. Nature 644 Genetics Reviews, 14, 807-820.

645 Shindo C., Aranzana M.J., Lister C., Baxter C., Nicholls C., Nordborg M., et al. (2005) Role 646 of FRIGIDA and FLOWERING LOCUS C in determining variation in flowering time of 647 Arabidopsis. Plant Physiology, 138, 1163-1173.

648 Simpson G.G., Dean C. (2002) Arabidopsis, the rosetta stone of flowering time? Science, 296, $649 \quad 285-289$.

650 Springthorpe V., Penfield S. (2015) Flowering time and seed dormancy control use external 651 coincidence to generate life history strategy. eLife, 4, e 05557.

652 Stinchcombe J.R., Weinig C., Ungerer M., Olsen K.M., Mays C., Halldorsdottir S.S., et al. 653 (2004) A latitudinal cline in flowering time in Arabidopsis thaliana modulated by the 654 flowering time gene FRIGIDA. Proceedings of the National Academy of Sciences, 101, $655 \quad 4712-4717$.

656 Suter L., Rüegg M., Zemp N., Hennig L., Widmer A. (2014) Gene regulatory variation 657 mediates flowering responses to vernalization along an altitudinal gradient in Arabidopsis. $658 \quad$ Plant Physiology, 166, 1928-1942. 
659 The 1001 Genomes Consortium (2016) 1,135 Genomes reveal the global pattern of 660 polymorphism in Arabidopsis thaliana. Cell, 166, 481-491.

661 Toorop P.E., Cuerva R.C., Begg G.S., Locardi B., Squire G.R., Iannetta P.P. (2012) Co662 adaptation of seed dormancy and flowering time in the arable weed Capsella bursa663 pastoris (shepherd's purse). Annals of Botany, 109, 481-489.

664 Tyagi A., Singh S., Mishra P., Singh A., Tripathi A.M., Jena S.N., et al. (2016) Genetic 665 diversity and population structure of Arabidopsis thaliana along an altitudinal gradient. $666 \quad A o B$ PLANTS, 8, plv145.

667 Vidigal D.S., Marques A.C.S.S., Willems L.A.J., Buijs G., Méndez-Vigo B., Hilhorst 668 H.W.M., et al. (2016) Altitudinal and climatic associations of seed dormancy and 669 flowering traits evidence adaptation of annual life cycle timing in Arabidopsis thaliana. $670 \quad$ Plant, Cell and Environment, 39, 1737-1748.

671 Weigel D. (2012) Natural variation in Arabidopsis: from molecular genetics to ecological 672 genomics. Plant Physiology, 158, 2-22.

673 Wilczek A.M., Roe J.L., Knapp M.C., Cooper M.D., Lopez-Gallego C., Martin L.J., et al. 674 (2009) Effects of genetic perturbation on seasonal life history plasticity. Science, 323, 930675934.

676 Wilczek A.M., Cooper M.D., Korves T.M. Schmitt J. (2014). Lagging adaptation to warming 677 climate in Arabidopsis thaliana. Proceedings of the National Academy of Sciences, 111, $678 \quad 7906-7913$. 
681 Fig. 1. (A) Map showing the geographical position of accessions indicating their GWR local 682 adjusted pseudo- $R^{2}$ values for DSDS50 and minimum temperature. (B) Map showing the 683 geographical position of accessions indicating their GWR local adjusted pseudo- $R^{2}$ values for 684 FT and minimum temperature.

685

686 Fig. 2. (A) Relationship between minimum temperature and the mean of the FT/DSDS50

687 ratio. (B) Relationship between minimum temperature and the standard deviation of the

688 FT/DSDS50 ratio. Regression lines are given for accessions below and above the turning

689 point at $7.2^{\circ} \mathrm{C}$, which maximises the $R^{2}$ values of the two groups of accessions. (C)

690 Relationship between minimum temperature and the correlation coefficients between FT and

691 DSDS50. Filled dots indicated significant correlation coefficients based on Dutilleul's

692 modified $t$ test. Regression lines are given for accessions with significant correlation

693 coefficients below and above the turning point at $7.3^{\circ} \mathrm{C}$, which maximises the $R^{2}$ values of the

694 two groups of accessions. In all three cases, values were obtained from temperature sliding

695 window analyses and ranked along the minimum temperature gradient.

696

697 Fig. 3. Minimum temperature map for the Iberian Peninsula indicating the geographical

698 position of $A$. thaliana populations with minimum temperatures below (hollow dots) and

699 above (filled dots) the turning poing of $7.3^{\circ} \mathrm{C}$ for the correlation between FT and DSDS50

700 along the minimum temperature gradient (see Fig. 2C).

701

702 


\section{SUPPORTING INFORMATION}

704

705 Fig. S1. (A) Relationship between minimum temperature and the correlation coefficients

706 between DSDS50 and minimum temperature. (B) Relationship between minimum

707 temperature and the correlation coefficients between FT and minimum temperature. Filled

708 dots indicated significant correlation coefficients based on Dutilleul's modified $t$ test. The

709 major turning point of each panel was detected at 7.4 and $6.9^{\circ} \mathrm{C}$ for DSDS50 and FT,

710 respectively. In all three cases, values were obtained from temperature sliding window

711 analyses and ranked along the minimum temperature gradient. 
(A)

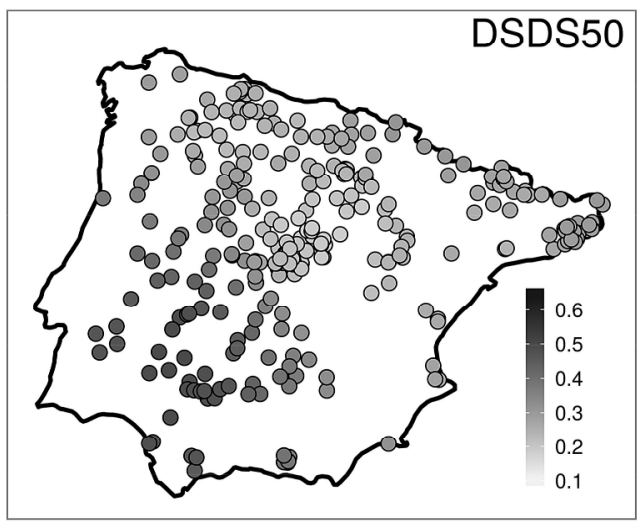

(B)

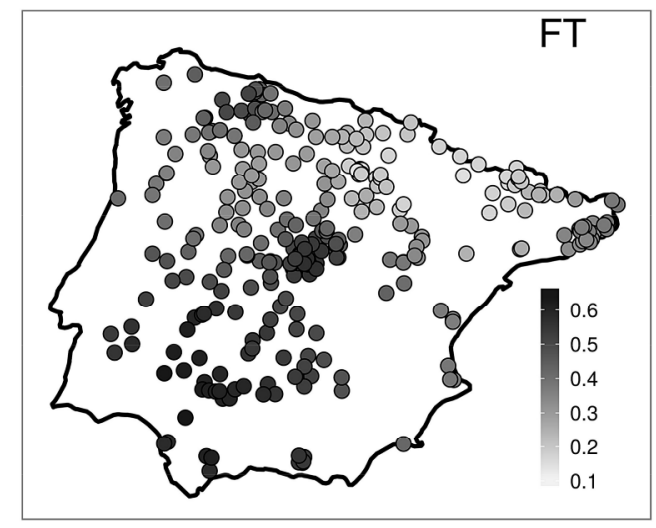

Fig. 1. (A) Map showing the geographical position of accessions indicating their GWR local adjusted pseudoR2 values for DSDS50 and minimum temperature. (B) Map showing the geographical position of accessions indicating their GWR local adjusted pseudo-R2 values for FT and minimum temperature.

Fig. 1

$297 \times 209 \mathrm{~mm}(300 \times 300$ DPI $)$ 
(A)

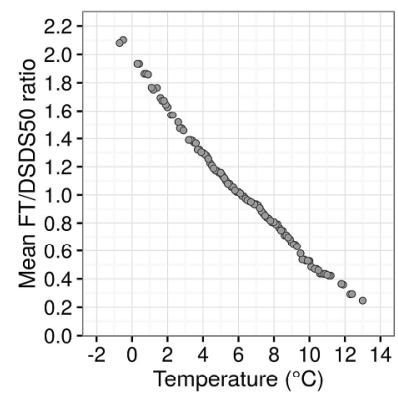

(B)

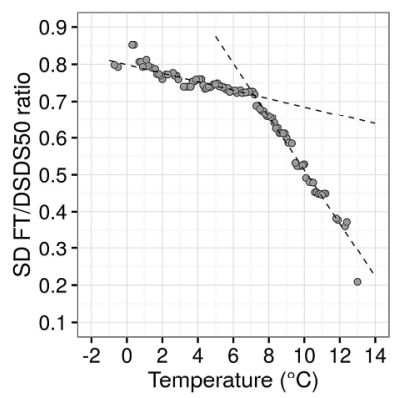

(C)

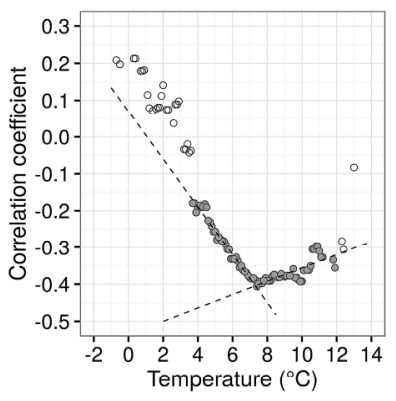

Fig. 2. (A) Relationship between minimum temperature and the mean of the FT/DSDS50 ratio. (B) Relationship between minimum temperature and the standard deviation of the FT/DSDS50 ratio. Regression lines are given for accessions below and above the turning point at $7.2^{\circ} \mathrm{C}$, which maximises the R2 values of the two groups of accessions. (C) Relationship between minimum temperature and the correlation coefficients between FT and DSDS50. Filled dots indicated significant correlation coefficients based on Dutilleul's modified t test. Regression lines are given for accessions with significant correlation coefficients below and above the turning point at $7.3^{\circ} \mathrm{C}$, which maximises the R2 values of the two groups of accessions. In all three cases, values were obtained from temperature sliding window analyses and ranked along the minimum temperature gradient.

Fig. 2

$297 \times 209 \mathrm{~mm}(300 \times 300 \mathrm{DPI})$ 


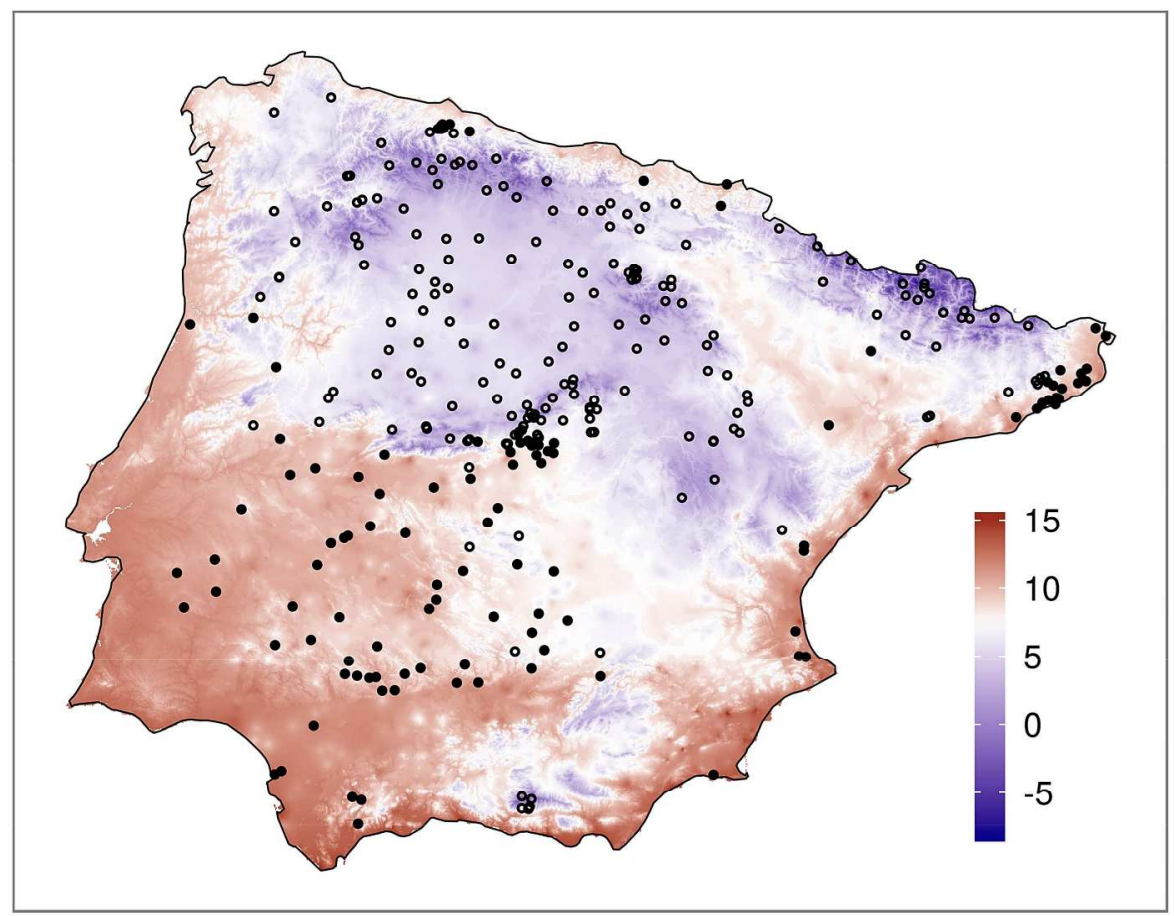

Fig. 3. Minimum temperature map for the Iberian Peninsula indicating the geographical position of A. thaliana populations with minimum temperatures below (hollow dots) and above (filled dots) the turning poing of $7.3^{\circ} \mathrm{C}$ for the correlation between FT and DSDS50 along the minimum temperature gradient (see Fig. 2C).

Fig. 3

$297 \times 209 \mathrm{~mm}(300 \times 300$ DPI $)$ 
(A)

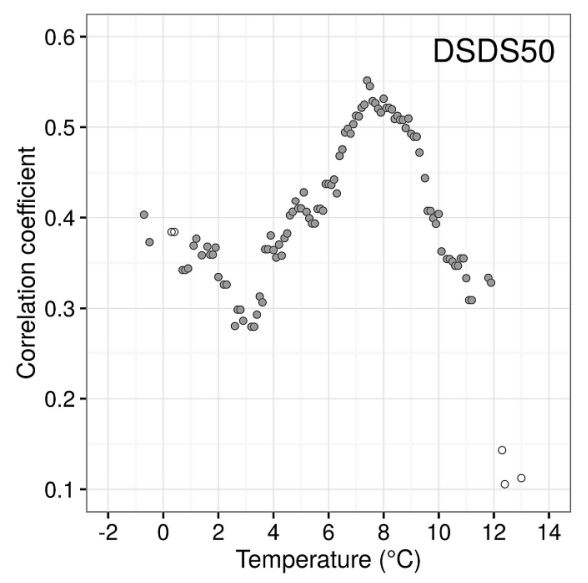

(B)

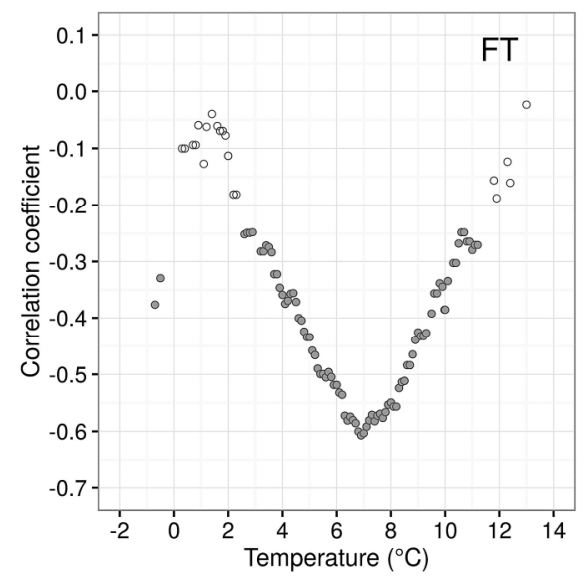

$297 \times 209 \mathrm{~mm}(300 \times 300$ DPI $)$ 